\title{
Managed Care - pro und kontra
}

Der Artikel von J. und M. Barben, in dem die Autoren auf der Basis eines Referats des US-Chirurgen John Nahas das Managed-Care-System US-amerikanischer Prägung kritisieren, hat eine Reihe teils ablehnender, teils zustimmender Reaktionen provoziert. Diese werden im folgenden als Schwerpunktthema im Sinne einer kontradiktorischen Diskussion wiedergegeben.

Die Redaktion

\section{Managed Care steigert die Qualität und senkt die Kosten der medizinischen Versorgung}

Felix Huber

* Barben J. Barben M. Gefährdet «Managed Care» die Behandlungsqualität? Schweiz. Ärztezeitung 2006;87(9):342-7.

Korrespondenz: Dr. med. Felix Huber Leiter MediX Zürich Rotbuchstrasse 46 CH-8037 Zürich
Der Artikel [1] fasst ein Referat von F. J. Nahas zusammen, der offensichtlich einen missionarischen Feldzug gegen Managed Care quer durch Europa unternommen hat. Die Ausführungen sind polarisierend und schildern die Verhältnisse in den USA sehr einseitig. Es haben sich zudem ein paar Vorwürfe eingeschlichen, die gar nichts mit Managed Care zu tun haben.

Die beklagten absurden Gerichtsprozesse wegen ärztlicher Kunstfehler mit astronomisch hohen Schadenersatzforderungen sind Ausdruck amerikanischer Rechtspraxis und der Haltung auf schonungslose Maximierung des persönlichen Gewinns in den USA. In den letzten Jahren waren davon auch Managed-Care-Organisationen betroffen (verweigerte Knochenmarktransplantation bei einer Frau mit metastasierendem Mammakarzinom). Die Haftung für hohe Schadenersatzsummen wird also nicht generell auf die Ärzte abgewälzt, und es betrifft in den USA alle Ärzte, unabhängig von Managed Care.

Im Artikel von Barben und Barben werden auch die Patientenverfügungen als typisches Beispiel einer Managed-Care-Medizin dargestellt, die in erster Linie Behandlungen verweigern wolle. Managed Care versucht, die Behandlung möglichst optimal $\mathrm{zu}$ koordinieren und die Patientenbedürfnisse dabei zu berücksichtigen. Dazu gehört auch die Frage nach einer Patientenverfügung. Sie wird bei uns in der Schweiz in zunehmendem Masse von verschiedener Seite gefördert (FMH, Patientenorganisationen, Dialog Ethik usw.). Patientenverfügungen sind kein Managed-Care-Spezifikum.

Viele erhobene Vorwürfe gegen Managed Care in den USA hängen mit dem dort existierenden
Versicherungssystem zusammen. Die Arbeitnehmer sind über die Firma kollektiv versichert, sie haben also auch keine Wahl über ihr Versicherungsprodukt. Die Unternehmen handeln mit Managed-Care-Organisationen Versicherungspläne aus und sind bestrebt, eine nachgewiesene hohe Qualität zu einem günstigen Preis einzukaufen. Das hat die Qualitätssicherung in der Medizin entscheidend gefördert. Das Problem der 45 Millionen US-Bürger, die keine Krankenversicherung haben, steht in keinem Zusammenhang mit Managed Care. Es sind Angestellte von kleinen Firmen, die nicht einem Versicherungsobligatorium unterliegen, Selbständige, die sich keine Krankenversicherung leisten können, oder Arbeitslose, die nicht unter Medicaid, die staatliche Versicherung für Mittellose, fallen.

Managed Care in der Schweiz entwickelt sich auf einem völlig anderen soziokulturellen Hintergrund. Die Versicherten wählen ihr Versicherungsprodukt frei und können sich aufs Jahresende wieder in die konventionelle Versicherung umteilen lassen. Managed Care kann sich in der Schweiz also nur mit einer laufend praktizierten hohen medizinischen Versorgungsqualität durchsetzen. Andernfalls würden die Versicherten solche Modelle nicht wählen. Tatsächlich ist es mit der mittlerweile 17jährigen Aufbauarbeit von Managed Care in der Schweiz gelungen, eine hohe Zufriedenheit bei den Patienten zu erreichen. Ein Beweis dafür sind die ständig steigenden Managed-Care-Versichertenzahlen in Hausarztmodellen und HMOs.

Managed Care in der Schweiz hat massgeblich zur Qualitätsdokumentation beigetragen. Ein Grossteil der HMOs und immer mehr Hausarzt- 
netze lassen sich EQUAM-zertifizieren. Das ist die strengste Qualitätskontrolle in der ambulanten Medizin. Managed-Care-Modelle konnten gleichzeitig nachweisen, dass sie beträchtliche Kosteneinsparungen von 10 bis $30 \%$ erreichen können.

Managed Care in der Schweiz bewegt sich in einem hochkompetitiven Umfeld. Auch in unserer Gesellschaft gibt es sehr unterschiedliche Erwartungshaltungen an die medizinische Versorgung. Dies verlangt nach einer entsprechenden Differenzierung auf der Angebotsseite der medizinischen Leistungserbringung. Die Versicherten sollen ihr Betreuungssystem wählen können, dafür unterschiedliche Preise bezahlen müssen und über die Entschädigung der Ärzte im gewählten Modell Bescheid wissen. Jedes Entschädigungssystem braucht Kontrollmechanismen und Absicherungsinstrumente, um Missbräuche einzugrenzen. Im Einzelleistungssystem muss man sich der Gefahr der Überversorgung, in Capitationmodellen des Risikos einer Unterversorgung bewusst sein. Wir haben diese Überlegungen wiederholt publiziert. Ärztliches Handeln kann letztlich nur in einer Atmosphäre des Vertrauens und in einer tragfähigen Arzt-Patienten-Beziehung erfolgreich sein.

\section{Literatur}

1 Barben J, Barben M. Gefährdet Managed Care die Behandlungsqualität? Schweiz Ärztezeitung 2006;87(9):342-7.

- Ashton CM. Hospital Use and Survival among Veteran Affairs Beneficiaries. N Engl J Med 2003; 349:1637-46.

- Baumberger J. So funktioniert Managed Care. Stuttgart: Thieme; 2001

- Baur R, Hunger W, Kämpf K, Stock J (Prognos AG). Evaluation neuer Formen der Krankenversicherung. Synthesebericht. Nr. 1/98. Beiträge zur sozialen Sicherheit. Bern: BSV; 1998.

- Hypertoniemanagement, Neue Formen der Krankenversicherung, Ein Praxisvergleich zwischen traditionellen Praxen und HMOs. Beiträge zur sozialen Sicherheit. Bern: BSV; 1998.

- Charta zur ärztlichen Berufsethik (European Federation of Internal Medicine, American College of Physicians, American Board of Internal Medicine). Schweiz Ärztezeitung 2003;84(45):2339 und 2347-9.
- Domenighetti G, Gutzwiller F, Martinoli S. Revisiting the most informed consumer of surgical services. Int J Techn Assess Health Care 1993; 4:505-13.

- Felder S, Werblow A. Mit Hausarztnetzen Kosten sparen. NZZ, 2. März 2004.

- Goetschi AS, Weber A. Ein Budget für Ärzte. Schweiz Ärztezeitung 2004;85(47):2498-503.

- Huber F. Managed Care in der Schweiz. Schweiz Ärztezeitung 2002;83(48):2629-32.

- Huber F. Verteilungsgerechtigkeit und Budgetverantwortung in Ärztenetzen. Schweiz Ärztezeitung 2004;85(35):1836-40.

- Hunter DJ. Getting a grip on clinical variations in hospital services. BMJ 2004;328:610.

- Lehmann H. Managed Care. Kosten senken mit alternativen Krankenversicherungsformen? Zürich: Rüegger; 2003.

- Marti C, Bovet U. Patientenverfügungen - eine Herausforderung für Patient und Arzt. Schweiz Ärztezeitung 2004;85(11):560-1.

- Moynihan R, Smith R. Too much medicine? Br Med J 2002;324:859-60.

- Pearson SD. Ethical guidelines for physician compensation based on capitation. N Engl J Med 1998;339:689-93.

- Pereira AG, Pearson SD. Patient attitudes toward physician financial incentives. Arch Intern Med 2001;161(10):1313-7.

- Roos A. Ethische Überlegungen zu Managed Care. Folia Bioethica 1999;50.

- Sommer J. Muddling Through Elegantly: Rationierung im Gesundheitswesen. Basel: EMH; 2001.

- Stoddard JJ, Grossmen JM, Rudell LS. Physicians more likely to face quality incentives than incentives that may restrain care. Issue Brief Cent Stud Health Syst Change 2002;(48):1-4.

- Weber A, Goetschi AS. Ärztenetze - die bessere Wahl. Schweiz Ärztezeitung 2003;84(44):2300-3.

- Welch H, Mogielnicki J. Presumed benefit: lessons from the American experience with marrow transplantation for breast cancer. Br Med J 2002; 324:1088-92.

- Wennberg JE. Use of hospitals, physician visits, and hospice care during last six months of life among cohorts loyal to highly respected hospitals in the United States. BMJ 2004;328:607-10.

- Zweifel P, Felder S. Demographische Alterung und Gesundheitskosten: Eine Fehlinterpretation. In: Oberender P (Hrsg.). Alter und Gesundheit. Gesundheitsökonomische Beiträge. Band 26. Baden-Baden: Nomos; 1999. 


\section{Managed Care bedeutet eine wirksame und erfolgreiche Stärkung der Grundversorger}

Thomas Lanter

Korrespondenz:

Dr. med. Thomas Lanter

SäntiMed

Ressort Medizinische Leitung

Kronenstrasse 22

CH-9230 Flawil
Leider stellt dieser Artikel keinen konstruktiven Beitrag zur Managed-Care-Diskussion dar. Bereits in der Einleitung wird von der Verfasserin erwähnt, dass die in der Folge geschilderten Verhältnisse im amerikanischen Gesundheitssystem mit den aktuellen Verhältnissen in der Schweiz kaum etwas gemeinsam haben. So ist dieser Artikel allenfalls als interessanter Beitrag über das amerikanische Gesundheitswesen $\mathrm{zu}$ beurteilen, er disqualifiziert sich aber bereits zu Beginn als Beitrag zu einer seriösen Diskussion über die Managed-Care-Entwicklung in der Schweiz. Die Schilderung des bekanntermassen grotesken amerikanischen Rechtssystems lässt einen schliesslich den letzten Zusammenhang zur Managed-Care-Entwicklung in unserem Land verlieren. In Schweizer Managed-Care-Kreisen besteht die klare Überzeugung, dass in den USA viele Fehler gemacht worden sind, und man ist bestrebt, diese hier zu vermeiden und daraus auch zu lernen. Das Hinterlistige an diesem Artikel ist der Umstand, dass durch den ganzen Text hindurch subtil suggeriert wird, diese beschriebenen amerikanischen Verhältnisse kämen so auch in der Schweiz vor. Entweder besitzt die Autorin erschreckend mangelhafte Kenntnisse über das schweizerische Managed-Care-Wesen oder es muss ihr der Versuch einer arglistigen Täuschung der Leserschaft vorgeworfen werden. Gewisse Aussagen (z. B. die Patienten sind einem Hausarzt fest zugeteilt, der als Gatekeeper darüber wachen muss, dass sie nicht $\mathrm{zu}$ viele medizinische Leistungen in Anspruch nehmen) grenzen, auf die Schweizer Managed-Care-Szene bezogen, bereits an Verleumdung!
Die Manged-Care-Landschaft in der Schweiz ist sehr lebendig und besitzt innovative, dynamische Ärztenetze, mit Ausnahme des Tessins, in allen Regionen der Schweiz. Sie zeigt aktuell ein starkes Wachstum (etwa 18\% der Schweizer Versicherten sind heute in einem mehr oder weniger gesteuerten Versicherungsmodell!).

Die in Netzwerken organisierten Ärzte versuchen eine qualitativ hochstehende Patientenversorgung durchzuführen. Dabei helfen ihnen Qualitätszirkel, in denen das medizinische Handeln kritisch reflektiert wird, interne Guidelines, Besprechungen von Überweisungen an Spezialisten und Spitäler, Critical Incidents Report, aber auch mündige Patienten, die bezüglich ihrer Gesundheitsprobleme gut aufgeklärt und informiert sind.

Managed Care bedeutet eine wirksame und erfolgreiche Stärkung der Grundversorger. Diese sind Garanten, dass die Patienten umsichtig im komplex gewordenen Gesundheitswesen geführt werden. Sie steuern die Behandlungsabläufe; und dies offenbar so erfolgreich, dass einige Versicherer ihren Kunden bis 15\% Prämienrabatt offerieren können.

Managed Care bedeutet daher auch eine starke und effiziente Alternative zur Auflösung des Kontrahierungszwanges.

Ich wünschte mir mehr Sachlichkeit in der Diskussion über Managed Care in der Schweiz. Solche polemischen Artikel, die gespickt sind mit Vorurteilen, führen zu gar nichts!

\section{Managed Care in der Schweiz und in den USA unterscheiden sich fast wie Tag und Nacht}

\section{Florian Suter}

Korrespondenz:

Dr. med. Florian Suter

Facharzt für Allgemeinmedizin FMH

Tannenstrasse 1

CH-4416 Bubendorf

Tel. 0619359222

Fax 0619359229

florian.suter@hin.ch
Mit anfänglichem Interesse, zunehmend aber mit etwas Stirnrunzeln, habe ich den Artikel «Gefährdet «Managed Care» die Behandlungsqualität?» in der SÄZ gelesen, in dem ein Vortrag des amerikanischen Managed-Care-Kritikers Frederick John Nahas zusammenfasst wird.

Ich habe mich dann immer wieder gefragt: Was wollen die Autoren mit ihrem Beitrag eigentlich? Ihre Absicht ist nicht klar deklariert, und bei einem derart komplexen Thema dienen schwammige Rahmenbedingungen nicht wirk- lich einer konstruktiven Auseinandersetzung. Wer, bitte, hat zum Beispiel das fettgedruckte Lead verfasst, in dem behauptet wird, die Autoren «möchten die Diskussion über diese Thematik anregen und vor einem schleichenden Übergang zu amerikanischen Verhältnissen warnen»? Frau und Herr Barben selbst? Ein SÄZ-Redaktor? Und wenn dort «vor einem schleichenden Übergang zu amerikanischen Verhältnissen» gewarnt wird - besteht denn ein Anlass zur Vermutung, ein solcher könnte drohen? Im selben Lead wird 
doch (meines Erachtens völlig zu Recht) festgehalten, dass sich «diese Erkenntnisse wohl nicht im Massstab 1:1 auf die hiesigen Verhältnisse übertragen lassen» - in welchem Massstab dann aber? 0,5:1? 0,1:1?

Wenn die Absicht des Artikels wirklich ist, die Diskussion über Managed Care im schweizerischen Gesundheitswesen anzuregen, weshalb folgt dann eine (soweit ich das beurteilen kann, berechtigte) seitenlange kritische Auseinandersetzung mit Managed Care in den USA? Und weshalb werden dann nicht Punkte aufgeführt, die den Autoren bei Managed Care im schweizerischen Gesundheitswesen kritisch erscheinen? In der vorliegenden Form wird, so jedenfalls wirkt der Beitrag auf mich, reichlich pauschal und eher unterschwellig vor Managed Care generell gewarnt, ohne konkrete Kritiken zu äussern.

Kennen die Autoren überhaupt Managed Care, wie es in der Schweiz praktiziert wird? Wissen sie etwa nicht, dass im System unserer sozialen Krankenversicherung, das sich weiss Gott und zum grossen Glück aller Beteiligten fast wie Tag und Nacht vom amerikanischen Krankenversicherungssystem (man müsste vielleicht eher von einem Kranken-Nicht-Versicherungssystem sprechen ...) unterscheidet, die meisten der geschilderten Missstände einfach nicht möglich sind [1]? Und ist ihnen nicht bekannt, dass ernst- hafte und verantwortungsbewusste Netzwerke mit Budgetverantwortung von Anfang an eine Zertifizierung z. B. bei der EQUAM-Stiftung (www. equam.org) anstreben; wer sich die Mühe nimmt (oder nähme), die EQUAM-Kriterien zu studieren, wird (oder würde) erkennen können, dass diese ganz wesentlich darauf aus sind, genau die in den USA zu Recht angeprangerten Missstände bei uns zu verhindern.

Ich komme leider zum Schluss, dass der Artikel gerade nicht einer konstruktiven Diskussion über Managed Care in der Schweiz förderlich ist, sondern die nach wie vor grosse Zahl meiner Berufskolleginnen/-kollegen, die - obwohl nicht über detaillierte Kenntnisse verfügend - Managed Care gleichsam als «des Teufels» ansehen und mithin eine ernsthafte Auseinandersetzung mit der Thematik nach wie vor verweigern, in ihrer bedauerlich engstirnigen Haltung bestärkt. Schade! Kein wirkliches Glanzstück von Frau und Herrn Barben - und bedauerlicherweise erst recht nicht von der Redaktion der SÄZ, die einen derart unausgewogenen Beitrag veröffentlicht.

1 Untermauert wird meine Aussage z.B. in einem ausführlichen Artikel in «The New York Review of Books» (Volume 53, Number 5) unter dem Titel «The Health Care Crisis and What to Do About It» (www.nybooks.com/articles/18802)

\section{Replik zu den Diskussionsbeiträgen der Befürworter von «Managed Care»}

\author{
Dr. phil. J. Barben, \\ Dr. med. M. Barben
}

Korrespondenz:

Dr. phil. Judith Barben Im Wuli 15

CH-8536 Hüttwilen
Das lebhafte, auch kontroverse Echo auf unseren Artikel «Gefährdet «Managed Care〉 die Behandlungsqualität?» zeigt, dass das Bedürfnis nach einer Diskussion gross ist. Mit unserem Bericht über die Vorträge des amerikanischen Chirurgen Nahas wollten wir ein Nachdenken über Managed Care anregen, weil das System im Zuge der weiteren KVG-Revision bei uns gesetzlich verankert werden soll [1].

Selbstverständlich kann man die amerikanische Situation nicht 1:1 auf die Schweiz übertragen, wo das Versicherungsmodell noch frei wählbar ist. Sicher ist es aber sinnvoll, aus den in Amerika gemachten Fehlern Schlüsse zu ziehen, um ähnliche Fehlentwicklungen bei uns zu verhindern. In den USA werden übrigens von einem einbezahlten Dollar nur gerade 13 Cents für medizinische Zwecke verwendet [1]. Die allermeisten Amerikaner, Versicherte und Ärzte, sind mit dem Managed-Care-System zunehmend unzufrieden. Die anfänglichen Versprechungen, dass mit Managed Care die Behandlungsqualität verbessert und Kosten gespart werden könnten, haben sich nicht erfüllt [3]. Natürlich hatte bei der Einführung des Systems in den USA noch niemand von Budgetverantwortung gesprochen. Diese wurde erst später eingeführt [4].

Wir sind froh, dass auch die hiesigen Vertreter des Managed-Care-Systems amerikanische Verhältnisse auf keinen Fall wollen. So weist beispielsweise der Leiter eines Ärztenetzwerkes auf die Gefahr einer medizinischen Unterversorgung durch das Kopfpauschalenmodell hin, eine Finanzierungsform von Managed Care, die bei uns in «Ärztenetzwerken mit Budgetverantwortung» bereits existiert [5]. Trotz eingehender Beschäftigung mit dem Schweizer Managed-CareSystem gelingt es uns nicht, darin irgendwelche Vorteile für die Patienten oder Ärzte zu erkennen. Denn der hauptsächliche, auch erklärte Sinn des Modells soll ja sein, Kosten einzusparen. Den Patienten kann das Modell nur mit Prämienverbilligungen schmackhaft gemacht werden, für die sie aber gravierende Einschränkungen in Kauf 
nehmen müssen. An diesen systemimmanenten Nachteilen können auch die Qualitätssicherungssysteme nichts ändern.

Bundesrat Couchepin und Hans Heinrich Brunner, vormals FMH-Präsident und BAG-Vizedirektor, heute im BAG zuständig für Sonderaufgaben wie Projekte zur Kostenkontrolle im Bereich der Krankenversicherung [6], wollen ebenfalls Kosten einsparen. Das ist ihr erklärtes und wichtigstes Ziel. H. H. Brunner hat bekanntgegeben, dass er auch in seiner neuen Position den Druck auf die Leistungserbringer aufrechterhalten und die Programme zur Kostensenkung vorantreiben will [7]. Mit Abstrichen im Leistungskatalog und Begrenzung der Anzahl der Anbieter (d.h. Aufhebung des Kontrahierungszwanges) will er Rationierungen durchführen, angeblich ohne Qualitätsverlust [8]. Vorerst sind Couchepin und Brunner mit ihrem Versuch, das Managed-Care-System gesetzlich zu verankern, im Parlament gescheitert. Doch sie bleiben auf diesem Weg. Um Widerstand zu vermeiden, gehen sie in kleinen Schritten vor. «Ein nicht zu harter Kurs» sei momentan besser, sonst drohe ein Referendum, meinte Brunner [8]. Die Aufhebung des Kontrahierungszwanges ist für ihn ein sinnvoller Schritt auf dem Weg zum Managed-Care-System [9], das möglicherweise in $\mathrm{Zu}$ kunft für alle verpflichtend und ohne Alternative sein soll.

Heute sind Krankenkassen noch verpflichtet, mit jedem Arzt zusammenzuarbeiten. Würde der Kontrahierungszwang aber aufgehoben, sähe die Situation ganz anders aus. Die Krankenkassen hätten dann die Macht, nur noch mit denjenigen Ärzten Verträge abzuschliessen, die «kostengünstig» arbeiten oder beispielsweise in ManagedCare-Netzwerken organisiert sind. Die Abschaffung des Kontrahierungszwanges wäre somit der Dammbruch, der in kurzer Zeit zu einer Zwei- klassenmedizin nach amerikanischem Vorbild führen könnte. In der Schweiz können sich heute - im Unterschied zu den meisten anderen Ländern - alle Menschen, ob arm oder reich, jung oder alt, eine gute medizinische Versorgung leisten. Die freie Arztwahl ist garantiert. Es ist uns ein grosses Anliegen, dieses gute und bewährte Gesundheitssystem zu erhalten. Wir sind davon überzeugt, dass die Mehrheit der Schweizer der Abschaffung einer guten medizinischen Versorgung und der freien Arztwahl nicht zustimmen wird, wenn sie richtig und vollumfänglich informiert wird.

\section{Literatur}

1 Neue Zürcher Zeitung, 16.9.2004: «Medizinische Netzwerke werden im Gesetz verankert, aber nicht obligatorisch (...) Seit dem Scheitern einer umfassenderen Revision der Krankenversicherung im Parlament geht der Bundesrat in kleinen Schritten vor.»

2 Nahas JF. Managed Care: Historical Perspective and its Impact on Patient Care. Vortrag in Zürich, 9. Dezember 2004

3 Nadolski H. Das Gesundheitssystem der USA. Eine Einführung. In: Angell M. Der Pharma-Bluff (The Truth about the Drug Companies). Bonn/ Bad Homburg: KomPart; 2005. S. 11.

4 Himmelstein D. Das US-Gesundheitswesen: Schrecken ohne Ende - und doch Vorbild für die Schweiz? Innenansicht eines Experten. Vortrag in Bern, 16. März 2005.

5 Baur HR. Kritische Bemerkungen zum Modell «Ärztenetzwerk mit Budgetverantwortung». Schweiz Ärztezeitung 2003;84(40):2081-2.

6 Mitteilung des Bundesamtes für Gesundheit. 7. Dezember 2005

7 NZZ am Sonntag, 11.12.2005.

8 Berner Zeitung, 13.10.2004.

9 «Sinnvoll sei die Vertragsfreiheit auch als Schritt zum nachweisbar kostensenkenden ManagedCare-Modell.» Berner Zeitung, 13.10.2004.

\section{Die prospektive Finanzierung medizinischer Leistungen gefährdet die Behandlungsqualität}

\section{David Winizki}

Korrespondenz:

Dr. med. David Winizki

Arzt für Allgemeinmedizin

Paulstrasse 11

CH-8008 Zürich
«Managed Care» - im Sinne der prospektiven Finanzierung medizinischer Leistungen - gefährdet die Behandlungsqualität. Das ist gar keine Frage, sondern ein realistisches Szenario, nur schon angesichts der evidenten Hinweise im Artikel von J. und M. Barben. Die Zustände im amerikanischen Gesundheitswesen sind sowohl für die Mehrzahl der Bevölkerung wie auch für die Mehrheit der Ärztinnen und Ärzte eine Schreckensvision. Ökonomische Zwänge dominieren aber das medizinische Handeln auch bei uns schon in einem Ausmass, wie sich das vor zehn Jahren noch niemand vorstellen konnte.

Capitationverträge, hausärztliche Netzwerke mit Budgetverantwortung, gibt es zum Beispiel bei uns bereits. Grundversicherte kriegen 10-20\% Prämienrabatt mit ihrem Beitritt zum Modell. Die Ärztinnen und Ärzte dürfen für diese Patienten/-innen zusammen nur für einen bestimmten Betrag Leistungen erbringen oder veranlassen. Diese Summe berechnet sich auf der Basis einer recht spekulativen Risikoberechnung. 
Gibt das ärztliche Kollektiv mehr aus, muss jedes Mitglied Ende Jahr solidarisch einen Teil zurückzahlen. Bleibt es unter dieser Summe, bekommt es eine Vergütung.

So weit, so gut. Aber wo ist jetzt die Gefährdung der Behandlungsqualität? Nehmen wir ein Beispiel: Ein dreissigköpfiges ärztliches Netzwerk handelte für insgesamt 1000 Versicherte einen Jahresplafond von einer Million Franken aus, Grossrisiken über 20000 Franken werden aus der Rechnung genommen. Im vergangenen Jahr wurde das Budget markant überschritten, und jedes Netzwerkmitglied musste 1000 Franken zurückzahlen. Einer der Kollegen hatte sich bei drei Prostatakarzinompatienten für eine DecapeptylBehandlung (5000 Franken/Jahr) entschieden. Im nächsten Jahr wird er - bei identischen Entscheidungsgrundlagen - zwei von drei Patienten die Kastration empfehlen, was viel günstiger ist.

Die Budgetverantwortung ist nicht nur für die medizinisch adäquate Versorgung der Grundversicherten eine Gefahr. Die Budgetverantwortung ist auch für uns Ärztinnen und Ärzte nicht lustig: Auf der einen Seite stehen wir unter dem Gruppendruck der Netzwerkkolleginnen/-kollegen, im nächsten Jahr nichts mehr zum Defizit beizutragen, auf der andern Seite verlieren wir das Vertrauen unserer Patienten/-innen. Es ist nämlich völlig illusorisch, zu meinen, in unsrer
Informationsgesellschaft würden diese nicht genau spüren, dass wir bei unsern Überlegungen dauernd die Kostenschere im Kopf haben.

Die einzigen, denen diese Ökonomisierung des Gesundheitswesens nicht schadet, sind jene, welche genug Geld haben, um die DecapeptylBehandlung aus der eigenen Tasche zu bezahlen. Sie werden sich sicher nicht kastrieren lassen. Zu ihnen gehören bestimmt alle jene Politiker, Krankenkassenmanager und Gesundheitsökonomen, welche uns diese Probleme einbrocken mit ihrer Forderung nach prospektiver Finanzierung der medizinischen Leistungen.

Ich finde, die sollen zuerst ihre Hausaufgaben im Bereich Beschäftigungs- und Sozialpolitik machen. Solange über 10\% der Bevölkerung im erwerbsfähigen Alter von Renten, Arbeitslosenoder Fürsorgegeld lebt und nochmals 10\% Working Poors sind, können wir Ärztinnen und Ärzte die Bevölkerung einfach nicht billiger behandeln. Armut macht krank, meine Herren Gesundheitspolitiker und Gesundheitsökonomen. Geben Sie sich doch bitte zuerst etwas mehr Mühe, eine Sozialpolitik zu machen, bei der nicht so viele Leute arm und krank werden, bevor sie uns Ärztinnen und Ärzte Vorschriften machen, was es kostet, das Geschirr zu flicken, das Sie dauernd kaputtmachen!

\section{«Managed Care» führt zu einer Verschlechterung der medizinischen Versorgung}

Gian Bischoff

Dr. med. Gian Bischoff

Vereinigung unabhängiger Ärzte

VUÄ

Postfach 2309

8031 Zürich

gian.bischoff@gmail.com
Man kann es drehen, wie man will: «Managed Care» führt zu einer Verschlechterung der medizinischen Versorgung, das zeigen die Ausführungen von J. und M. Barben eindrücklich. Wie könnte es denn auch anders sein, wenn anstelle von medizinischen Überlegungen finanzielle Anreize gesetzt werden! Und dies kann wohl nicht genügend betont werden, gegen alle Propagandamühlen von Krankenkassen und anderen wirtschaftlich Profit daraus Schlagenden.

Der gesunde Menschenverstand müsste uns eigentlich schon lehren, dass jedes Modell prospektiver Finanzierung die medizinische Entscheidungsfindung beeinflusst. Die Art und Weise, wie dies im Einzelfall umgesetzt wird, ist weniger entscheidend wie das grundsätzliche Problem, das dem Modell inhärent ist. Wenn von vornherein feststeht, wieviel Geld für die Behandlung meiner Patienten zur Verfügung steht, unabhängig davon, wie ich sie behandle, dann ist der Profit um so grösser, je weniger Geld ich für sie ausgebe. In den gegenwärtig umgesetzten
Modellen mögen die Sanktionen (bei Überschreiten der vereinbarten Kosten) oder Belohnungen (bei entsprechendem Unterschreiten) noch wenig spürbar sein, die Tendenz bleibt dieselbe und die Auswirkungen eines solchen Modells, ist es einmal flächendeckend eingeführt, in gleicher Weise absehbar. Denn: Unvermeidlich ob bewusst oder unbewusst, ob ein bisschen oder ein bisschen mehr - werde ich mir bei jeder teuren Behandlung einmal mehr überlegen, ob ich sie wirklich verschreiben will. «Das ist ja genau das, was wir wollen! Damit werden ja gerade unnötige Behandlungen vermieden!» werden die Propagandisten von Managed Care rufen, nur: Unterlasse ich sie tatsächlich, weil sie unnötig ist? Oder doch nur, weil sie mein Budget zu sehr belasten könnte? Weil ich nicht das schwarze Schaf in meinem Capitationnetzwerk sein will, das daran schuld ist, dass alle Geld zurückzahlen müssen?

Wer unterscheidet zwischen unnötigen und notwendigen Behandlungen? Gibt es dazu an- 
dere als rein medizinische Kriterien (Medizin im umfassendsten Sinne gemeint)? Und wird der finanzielle Druck meine medizinischen Überlegungen verbessern (wie uns suggeriert wird) oder aber meine Ethik vergiften? J. und M. Barben zeigen eindrücklich, was es für Möglichkeiten gibt, Druck auf die ärztliche Entscheidungsfindung auszuüben (Angst vor Ausschluss aus der Versicherung, Angst vor finanziellem Regress, Angst vor Klagen ...). Alle diese Mechanismen untergraben letztlich die Medizin, sie alle führen dazu, dass medizinische Entscheidungen nicht nach Regeln der Kunst, sondern nach Massgabe der Rentabilität getroffen werden.

Die Konsequenzen für die medizinische Grundversorgung sind unabsehbar: Können Patienten noch Vertrauen haben zu ihrem Arzt, wenn sie wissen, dass sein Gehalt davon abhängt, wieviel Kosten sie verursachen? Werden sie ihm glauben, wenn er ihnen vorschlägt, vorerst zuzuwarten und auf eine Behandlung zu verzichten? Werden sie gesünder werden, wenn jeder Gang zum Arzt von Misstrauen geprägt ist?

Und wird die Medizin dadurch billiger, wie man uns glauben machen will? Diejenigen, welche schlechter behandelt werden, werden zwar weniger Kosten generieren, da ihnen ja medizinische Leistungen vorenthalten werden. Das werden diejenigen sein, welche sich eine private Zusatzversicherung nicht leisten oder die Behandlung nicht selber bezahlen können. Das sind dieselben, welche das grösste Risiko haben, chronisch krank zu werden, das grösste Risiko haben, arbeitsunfähig zu werden, das grösste Risiko haben, sozialfürsorgeabhängig zu werden. Die Kosten werden damit letztlich einfach verschoben - im Grunde genommen die einzige momentan praktizierte Methode, um Kosten (vermeintlich!) zu senken ...

Und was geschieht mit denen, die es sich leisten können? Sie werden sich, wenn irgend möglich, eine Zusatzversicherung leisten. Dies ist letztendlich die eigentliche Motivation, welche hinter den Konzepten von Managed Care u.ä. steckt: Verschlechterung der Grundversorgung für die Allgemeinheit und damit Erzeugung eines grösstmöglichen Anreizes (um nicht $\mathrm{zu}$ sagen Druckes), sich anderweitig zu versichern, da lediglich die Zusatzversicherungen sich dazu eignen, Profite zu generieren. Hand aufs Herz, gibt es für diese «Reformen» irgendeinen anderen ersichtlichen Grund?

Es wird uns gesagt, es ginge ums Sparen, die Gesundheitskosten würden ins Unermessliche steigen. Gleichzeitig wissen wir, dass es nicht die Gesundheitskosten sind, welche explodieren, sondern die Krankenkassenprämien, weil sich der durch Steuersenkungen ausgehungerte Staat aus der Finanzierung des Gesundheitswesens zurückzieht. Wir wissen auch, dass dasjenige Gesundheitssystem das teuerste der Welt ist, das uns Konzepte wie Managed Care, prospektive Finanzierung usw. verkaufen will.

Sparen könnte man auf andere Weise durchaus - wenn man das wirklich wollte -, nur eben daraus keinen Profit schlagen. Ärztenetzwerke wären durchaus sinnvolle Möglichkeiten einer effektiven Zusammenarbeit im Bereich der Grundversorgung, aber nur ohne Budgetverantwortung. Gatekeeper-Modelle würden durchaus Sinn machen, aber eben nur in finanzieller Unabhängigkeit! Die Frage ist nur, wer daran ein Interesse hat ...

\section{Weitere Zuschriften zum Artikel «Gefährdet «Managed Care» die Behandlungsqualität?»}

\section{Kosteneinsparungen sind auch anders möglich}

Der Artikel hat mich nachdenklich gemacht. Ich war an und für sich teilweise dem System «Managed Care» positiv eingestellt aufgrund von persönlichen Kontakten mit gewissen Vertretern ärztlicherseits, aber auch mit Kostenträgern. Dabei handelt es sich jedoch um Berufskollegen, die mir ihr Konzept im Sinne einer fachübergreifenden Zusammenarbeit mindestens teilweise plausibel machen konnten.
Anderseits hat nun diese amerikanische Darstellung für amerikanische Verhältnisse mir sehr zu denken gegeben. Sie haben sich als sehr extrem erwiesen und sind in einer Art für unser System in der Schweiz ungünstig, indem nicht nur die freie Arztwahl extrem eingeschränkt wird, sondern mit dem Capitationprinzip ein System zur Diskussion steht resp. eingeführt wurde, mit dem ich mich wirklich gar nicht einverstanden erklären kann. 
Dass ein solches System kostensenkend sein kann, ist mir klar. Es geht hier aber aus meiner Sicht rein um Kosteneinsparungen, primär ausgehend von Kostenträgern selbst und nicht um eine Verbesserung der Betreuung und Behandlung unserer Patienten, für die wir ja an und für sich da sind. Ich kann mir vorstellen, dass wertvolle Kosteneinsparungen gemacht werden können mit anderen Systemen, die die Zusammenarbeit jedoch zwischen Patient, Leistungserbringer und Kostenträger viel besser darstellen könnten, nämlich ein durchdachtes und konsequent durchgeführtes Fallmanagement. Da habe ich viel bessere persönliche Erfahrung gemacht, Voraussetzung jedoch ist, dass das System durchdacht und auch personell gut bestückt ist.

\section{Dr. med. Otto Knüsel, Valens}

\section{Sensibilisierung für ungute Entwicklungen}

Für die sehr klaren und genauen Ausführungen des Managed-Care-Modells in den USA möchte ich mich sehr bedanken.

Es wird deutlich, dass das gegenwärtige amerikanische System der «gemanagten» Gesundheitsfürsorge ein rein profitorientiertes Gesundheitswesen darstellt. Vom Arzt wird verlangt, dass er als eine Art Torhüter agiert, der über Zuteilung oder Ablehnung einer Krankenversorgung befindet. Die Fürsorge soll dem Profit dienen. Wir Ärzte werden unserer beruflichen Zuständigkeit beraubt, die Patienten ihrer Persönlichkeit. Der Artikel ist ein Beitrag zur Sensibilisierung im Umgang mit den Entwicklungen in der Schweiz und auch anderen europäischen Ländern.

\section{Birgit Grimm, Winterthur}

\section{Patientenverfügungen und «Managed Care»}

Patientenverfügungen dienen, entgegen der Behauptung der Autoren [1], genau diesem Zweck: der Achtung vor der Selbstbestimmung des einzelnen Individuums, dessen Wille zu respektieren unsere Pflicht ist.

Wintimed, ein seit Jahren erfahrenes Hausarztnetz, hat sich intensiv mit dem Thema Patientenverfügung auseinandergesetzt und auch im Rahmen einer öffentlichen Patientenveranstaltung, welche auf grosses Interesse stiess, sehr gute und positive Erfahrungen gemacht. So wurde unter anderem eine Dokumentation erarbeitet, welche als Grundlage eines problem- bezogenen Gespräches zwischen Hausarzt und Patient dient und diesem hilft, sich zu orientieren und seinen Willen, im Falle einer schwersten Erkrankung, quasi testamentarisch, kundzutun. Es ist grotesk, zu behaupten, Patientenverfügungen seien ein Mittel, um auf dem Rücken des Patienten Kosten zu sparen.

Niemand drängt hier den Patienten eine Verfügung auf, im Gegenteil, wir werden danach gefragt. Patienten sind mündige Personen, die genau verstehen, worum es bei einer Patientenverfügung geht und wozu sie dient. Sie entlastet in schweren Stunden auch Angehörige und Ärzte als wichtige Entscheidungshilfe für oder gegen eine Therapie im Sinne des Betroffenen und hat auch nicht den Zweck, eine palliative Behandlung zu verhindern.

In unserer demokratischen Gesellschaft ist der einzelne noch in der Lage, zu entscheiden, welchen Weg er im Sterben gehen will. Wir beraten ihn dabei mit Respekt und ohne Machbarkeitswahn. Wir dürfen noch verfügen, auch als $\mathrm{Pa}-$ tienten, zum Glück.

Dr. med. Ursula Bovet-Charles, Ärztin für Allgemeinmedizin FMH und Mitglied Wintimed, Winterthur

\section{Gefährdet «Managed Care» die Behandlungsqualität?}

Durch den Artikel von J. und M. Barben ist die Kontroverse um das Versicherungsmodell «Ärztenetzwerke mit Budgetverantwortung» und «Capitation» neu aufgeworfen worden. Befürworter dieser Modelle haben in letzter Zeit mit fast missionarischem Eifer und unter Zuhilfenahme von Medizinjournalisten versucht, in dieser Zeitung eine breite Ärzteschaft von den Vorteilen dieser Modelle zu überzeugen, welche nach Ansicht der Autoren auch in bezug auf die Qualität der ärztlichen Versorgung und Fortbildung wesentliche Vorteile mit sich bringen würden [1].

Gute ärztliche Fortbildung und Qualitätskontrolle haben nun wirklich nichts mit ärztlicher Budgetverantwortung zu tun und werden seit Jahren mit Erfolg auch in lockeren ärztlichen Zirkeln praktiziert, ohne Einbindung in eine Managed-Care-Organisation.

Meine Bedenken und Einwände gegenüber einer Einbindung des Arztes in die finanziellen Folgen seiner Diagnostik und Therapie sind seit meiner Stellungnahme in dieser Zeitung und in der NZZ die gleichen geblieben und sollen hier nicht alle repetiert werden [2-4]. 
Es ist nicht gut, wenn persönliche finanzielle Überlegungen des Arztes das Vertrauensverhältnis Arzt-Patient belasten und ärztliche Entscheidungen gerade bei aufwendiger Diagnostik und Therapie wie in der ambulanten Onkologie (PET, sehr teure Medikamente) von solchen Überlegungen beeinflusst werden können.

Es ist besser, wenn sich der Arzt zusammen mit dem Patienten frei für die beste Diagnostik- und Therapiestrategie entscheiden kann, ohne persönliche finanzielle Risiken. Diese sind nach Ansicht der Medix-Ärzte zwar «nie existenzgefährdend». (Aber doch genügend gross, um unter Umständen Risse und Gräben im Arzt-PatientenVerhältnis zu erzeugen.)

Es ist sicher nichts dagegen einzuwenden, dass Capitationmodelle auf dem Versicherungsmarkt angeboten werden. Der Einbezug des behandelnden Arztes in das finanzielle Risiko muss aber dem potentiellen Kunden klar und nicht nur im Kleingedruckten mitgeteilt werden, bevor er durch ein besonders günstiges Angebot angeworben wird. Wieviel durch solche Modelle tatsächlich gespart wird, kann nicht durch harte Daten belegt werden, da naturgemäss v. a. junge, gesunde Patienten diese Modelle wählen.

Kostengünstige Medizin kann auch durch Zusammenschluss in lockeren Ärztenetzwerken ohne Budgetverantwortung und durch enge $\mathrm{Zu}$ sammenarbeit mit einem kostengünstigen Spital erreicht werden!

Aus dem Artikel von J. und M. Barben geht deutlich hervor, dass amerikanische und nicht schweizerische Verhältnisse drastisch geschil- dert werden. Sicher liefert unser soziales Versicherungssystem ein gewisses Bollwerk gegen solch übertriebene Praktiken. Dennoch wissen wir, dass gewisse Ideen v. a. bei Krankenkassen, oft auf guten Boden fallen und dass die potentiellen Gefahren dieser Praktiken frühzeitig analysiert und bekanntgemacht werden müssen. Dass diese Gedanken nicht abwegig sind, soll eine persönliche Erfahrung vom Februar 2006 illustrieren: Ein Casemanager einer grossen Krankenkasse (mit sehr knappen medizinischen Kenntnissen) versuchte, die Familie eines Privatpatienten mit schwerster Herzinsuffizienz und chronischem Lungenödem, hospitalisiert in einem auswärtigen Spital, zu überzeugen, direkt in eine Rehabilitationsklinik überzutreten. Eine vorherige Therapieeinstellung in einer kardiologischen Abteilung erachtete er als nicht notwendig! Die Gefahr, dass medizinische Laien, welche rein finanzielle Ziele verfolgen, die Qualität unserer ärztlichen Behandlung beeinflussen, ist real!

Prof. Dr. med. H. R. Baur, Klinikleiter Medizinische Klinik, Spital Bern Tiefenau

1 Stricker B. «Ärztenetzwerke»-Klärung eines Begriffs. Schweiz Ärztezeitung 2005;86(15):883-7.

2 Baur HR. Kritische Bemerkungen zum Modell «Ärztenetzwerk mit Budgetverantwortung». Schweiz Ärztezeitung 2003;84(40):2081-2.

3 Baur HR. Ärzte mit Verantwortung Schweiz Ärztezeitung 2003;84(50):2652.

4 Baur HR. NZZ vom 26. Mai 2004. 